\title{
Propagation of waves above a plage as observed by IRIS and SDO
}

\author{
P. Kayshap ${ }^{1}$, A. K. Srivastava ${ }^{2}$, S. K. Tiwari ${ }^{3,4}$, P. Jelínek ${ }^{1}$, and M. Mathioudakis ${ }^{5}$ \\ 1 University of South Bohemia, Faculty of Science, Institute of Physics, Branišovská 1760, 37005 České Budějovice, Czech Republic \\ e-mail: virat.com@gmail.com \\ 2 Department of Physics, Indian Institute of Technology (BHU), Varanasi, India \\ 3 Lockheed Martin Solar and Astrophysics Laboratory, 3251 Hanover Street, Building 252, Palo Alto, CA 94304, USA \\ 4 Bay Area Environmental Research Institute, NASA Research Park, Moffett Field, CA 94035, USA \\ 5 Astrophysics Research Centre, School of Mathematics and Physics, Queen's Univeristy, Belfast BT7 1NN, UK
}

Received 11 June 2019 / Accepted 15 October 2019

\begin{abstract}
Context. Magnetohydrodynamic waves are proposed as the mechanism that transport sufficient energy from the photosphere to heat the transition region (TR) and corona. However, various aspects of these waves, such as their nature, propagation characteristics, and role in the atmospheric heating process, remain poorly understood and require further investigation.

Aims. We aim to investigate wave propagation within an active-region plage using IRIS and AIA observations. The main motivation is to understand the relationship between photospheric and TR oscillations. We identify the locations in the plage region where magnetic flux tubes are essentially vertical, and further we discuss the propagation and nature of these waves.

Methods. We used photospheric observations from AIA (i.e., AIA $1700 \AA$ ) as well as TR imaging observations (IRIS SJI Si IV $1400.0 \AA$ ). We investigated the propagation of the waves into the TR from the photosphere using wavelet analysis (e.g., cross power, coherence, and phase difference) with the inclusion of a customized noise model.

Results. A fast Fourier transform algorithm shows the distribution of wave power at photospheric and TR heights. Waves with periods between 2.0 and $9.0 \mathrm{~min}$ appear to be correlated between the photosphere and TR. We exploited a customized noise model to estimate the $95 \%$ confidence levels for the IRIS observations. On the basis of the sound speed in the TR and estimated propagation speed, these waves are best interpreted as slow magneto acoustic waves (SMAWs). It is found that almost all locations show correlation and propagation of waves over a broad range of periods from the photosphere to the TR. Our observations suggest that the SMAWs spatial occurrence frequency is stronly correlated between the photosphere and transition region within plage areas.
\end{abstract}

Key words. Sun: oscillations - Sun: faculae, plages

\section{Introduction}

Understanding wave propagation is a very important topic in solar physics as these waves can transport energy into the upper layers of the Sun's atmosphere. The energies carried by these waves can play a crucial role in the heating of the interface region and inner corona. The heating of the interface region cannot be fully understood without the characterization of wave propagation and the effect of the complex solar atmosphere on it. Plasma conditions, perturbations, and structured magnetic fields lead to a complex behavior of waves in the magnetic flux tube. There are several reports that use observations from different instruments and numerical simulations, and they shed light on various aspects of the waves (e.g., origin, properties, and dynamics) and their propagation throughout the solar atmosphere (De Moortel et al. 2000, 2002; De Pontieu et al. 2003; Centeno et al. 2006, 2009; Jelínek \& Karlický 2009; Jess et al. 2012; Jelínek \& Murawski 2013; Jelínek et al. 2015; Krishna Prasad et al. 2015; Murawski et al. 2018; Kayshap et al. 2018). In sunspot umbra, 3 min waves propagate up to the chromosphere from the photosphere, as reported by Centeno et al. (2006, 2009) using spectropolarimetric observations. InterfaceRegion Imaging Spectrometer (IRIS) observations show that the 3 min oscillations can propagate within the umbra up to the TR and corona (Tian et al. 2014; Khomenko \& Collados 2015). In magnetic free regions (i.e., inter-network), the propagation of 3 min waves is widely reported (e.g., Lites et al. 1982; Wikstøl et al. 2000; Judge et al. 2001; Bloomfield et al. 2004; Kayshap et al. 2018). Using IRIS high-resolution observations, Kayshap et al. (2018) explored the propagation of $3 \mathrm{~min}$ oscillations in the inter-network. The propagation of low frequencies (5 min) from the photosphere to the higher layers is also reported in a small magnetic patch by Srivastava et al. (2008).

In coronal loops, De Moortel et al. (2002) have shown that the photospheric oscillations (specifically, 3 and $5 \mathrm{~min}$ ) can reach up to the TR and coronal heights. It has been proposed that these are different manifestations of SMAWs in coronal loops (Jess et al. 2012; Krishna Prasad et al. 2015). Using TRACE observations, it has also been reported that photospheric power ( $p$-mode) can reach the TR within the plage atmosphere (De Pontieu et al. 2005) and the interaction of $p$-mode with the magnetic field generates magnetohydrodynamic wave modes (Spruit 1991; Cally et al. 1994; Jess et al. 2015). The magnetic field acts as a guide for the waves to reach up to coronal heights (Cally 2007; De Pontieu et al. 2003, 2005).

Almost all the works that investigate wave propagation use wavelet analysis for the study of phase relations. The first and most basic necessity has to do with the reliability of the wave periods that are detected through wavelet analysis. The reliability of the period depends on the confidence levels that are measured with the help of the assumed theoretical spectrum. The white noise (i.e., flat spectrum with no frequency dependency) 
is the most widely used theoretical spectrum to calculate the confidence levels. However, white noise does not represent the true noise inherited in the signal of the solar atmospheric. The use of an incorrect noise model may lead to the false detection of wave periods (Auchère et al. 2016; Threlfall et al. 2017). It has been reported that the Fourier spectra of coronal signals behave as power laws (e.g., Auchère et al. 2014; Gupta 2014; Inglis et al. 2015; Ireland et al. 2015). The power law nature of Fourier or wavelet spectra (i.e., $P \propto f^{-\alpha}$ ) is characteristic of red noise when the exponent value is -2 . The exponent values may vary from one time series (TS) to another. Therefore, we emphasize the importance and necessity of the power-law model for the estimation of the confidence levels (e.g., Gabriel et al. 2002; Vaughan 2005; Auchère et al. 2016; Pugh et al. 2017). Finally, we note that Auchère et al. (2016) beautifully justify the necessity of using a power law (along with kappa function and constant background) to detect wave periods in coronal loops. Our work follows Auchère et al. (2016) method of calculating the confidence levels using a power law function.

The main motivation of our work is to identify the nature of wave motions and their propagation properties in an activeregion plage area using high-resolution observations from IRIS (De Pontieu et al. 2014). The work is organized as follows. Section 2 describes the observational data and analysis. Section 3 describes the deduced results under three different subsections: Sect. 3.1 is dedicated to Fourier maps of plages in the different frequency bins; Sect. 3.2 describes the wavelet analysis and associated results using SDO/AIA $1700 \AA$ (Lemen et al. 2012) and IRIS SJI $1400 \AA$; Sect. 3.3 presents our investigation of the nature of SMAW waves in the plage region. In Sect. 4 the discussion and conclusions are outlined. In addition, Appendix A presents the diagnostics which show that Si IV line emission dominates the IRIS SJI $1400 \AA$ channel.

\section{Observational data and $A$ analysis}

IRIS observed an active-region plage on 28 July 2014 from approximately 17:59 UT to 19:52 UT. IRIS captures the solar spectra in the near- and far-ultraviolet, which includes many photospheric and chromospheric-TR lines (e.g., Mn I 2803.8 $\AA$, Mg II k $2796 \AA$, C II $1334.53 \AA$, Si IV $1393.75 \AA$ ). In this study we used mainly the IRIS/Slit-Jaw Imager (SJI) at $1400 \AA$, Si IV $1393.75 \AA$, and AIA $1700 \AA$. In addition, we also utilized magnetic field inclination and line of sight (LOS) magnetic field from HMI. At the time of the observations, the active-region (AR) plage was located at disk center (i.e., very close to $\mu=1.0$ ). The on-disk observations minimize projection effects (Falconer et al. 2016). However, another possibility of projection effects is due to the inclination of the magnetic field, which can lead to offsets at different heights.

AIA $1700 \AA$ imaging observations sample the emission from the upper photosphere, while IRIS SJI Si IV $1400 \AA$ is dominated by a spatially varying mix of upper photospheric continuum and low TR emission. The IRIS SJI $1400 \AA$ filter is a broadband filter with a width of $55 \AA$, and is dominated by continuum emission in the vicinity of two strong Si IV lines. Therefore, this filter can also capture some photospheric emission. In order to check the parts of the atmosphere that contribute to the emission in the IRIS SJI (i.e., photosphere or TR), we investigated the IRIS SJI $1400 \AA$ observations in conjunction with Si IV. Using this analysis we found that the IRIS SJI 1400 filter is dominated by the TR emission in the plage area. However, we did not find a well-defined correlation between SJI 1400 and the Si IV line in the surrounding quiet-Sun (QS) region. This suggests that our region of interest (ROI) is dominated by the TR emission. More information is provided in Appendix A.

Figure 1 shows AIA $1700 \AA$ (panel a) and IRIS SJI $1400 \AA$ (panel b) images of the observed plage region. We also show the LOS photospheric magnetic field (panel c) and field inclination angles (panel d). Strong magnetic fields are evident in the vicinity of the plage. The inclination angle from these plage locations varies from zero (i.e., vertical magnetic field in the central areas) to $70-80^{\circ}$ (at the outer edges). However, we are interested in the vertical magnetic field locations within these plage areas. Therefore, we put a threshold of magnetic field (i.e., higher than $300 \mathrm{G}$ ) to identify the vertical magnetic field plage locations (VMPLs). The identified plage locations are overdrawn by green contours on the inclination map (panel d). The inclination angles are very low within the VMPLs, which justifies that the magnetic field is essentially vertical. After identification of VMPLs, we extracted the TS from AIA $1700 \AA$ and IRIS SJI $1400 \AA$ data.

\section{Results}

We investigated wave propagation between the photosphere and the TR in the VMPLs. We utilized wavelet transform (e.g., cross wavelet transform, coherence, and phase difference) for this work. However, before presenting the wavelet analysis and the associated results, we present Fourier power maps of the plage region. This provides the wave power distribution within the plage region at two different heights (i.e., AIA $1700 \AA$ and IRIS $1400 \AA$ ).

\subsection{Fourier maps of the plage}

A fast Fourier transform (FFT) of the plage observations is performed in AIA $1700 \AA$ (i.e., photosphere) and IRIS $1400 \AA$ (TR). The FFT provides the power spectral density (i.e., wave power versus frequency) over a particular solar region (e.g., plage) in the present work. We normalized the Fourier power as explained in Gabriel et al. (2002), and used by Froment et al. (2015) and Auchère et al. (2016). Based on this methodology we estimated the scaling factor $(m$, as defined in previous works) corresponding to the confidence levels used. We estimated the scaling factor value to be 8.41 for confidence levels of $95 \%$,i.e., if the power is higher than 8.41 then the confidence level is above $95 \%)$. Using the value of the scaling factor $(m)$ and estimated noise we derived the final array ( $m *$ estimated noise) to normalize the Fourier power for each pixel. Finally, we estimated the normalized power maps for each frequency at each location in the observed region. In order to highlight the wave power distribution in the observed region, we selected three different frequency ranges: (1) the high-frequency range $-11.04 \mathrm{mHz}$ (period: $1.5 \mathrm{~min}$ ), (2) the intermediate-frequency range $-5.31 \mathrm{mHz}$ (period: $3.10 \mathrm{~min}$ ), and (3) the low-frequency range $-1.64 \mathrm{mHz}$ (periods: $10.15 \mathrm{~min}$ ). We extracted 2D Fourier maps (for these three selected frequencies) for AIA $1700 \AA$ and IRIS $1400 \AA$. The 2D Fourier maps for both channels are shown in Fig. 2.

High frequency. In Fig. 2, we show the Fourier power map in the high-frequency range (i.e., $11.04 \mathrm{mHz}$ ) for AIA $1700 \AA$ (panel a) and IRIS $1400 \AA$ (panel b). The observed area contains mostly a plage region along with the surrounding region at the edges of images (see Fig. 1). The normalized Fourier power maps show uniform distribution of the power of the waves in 


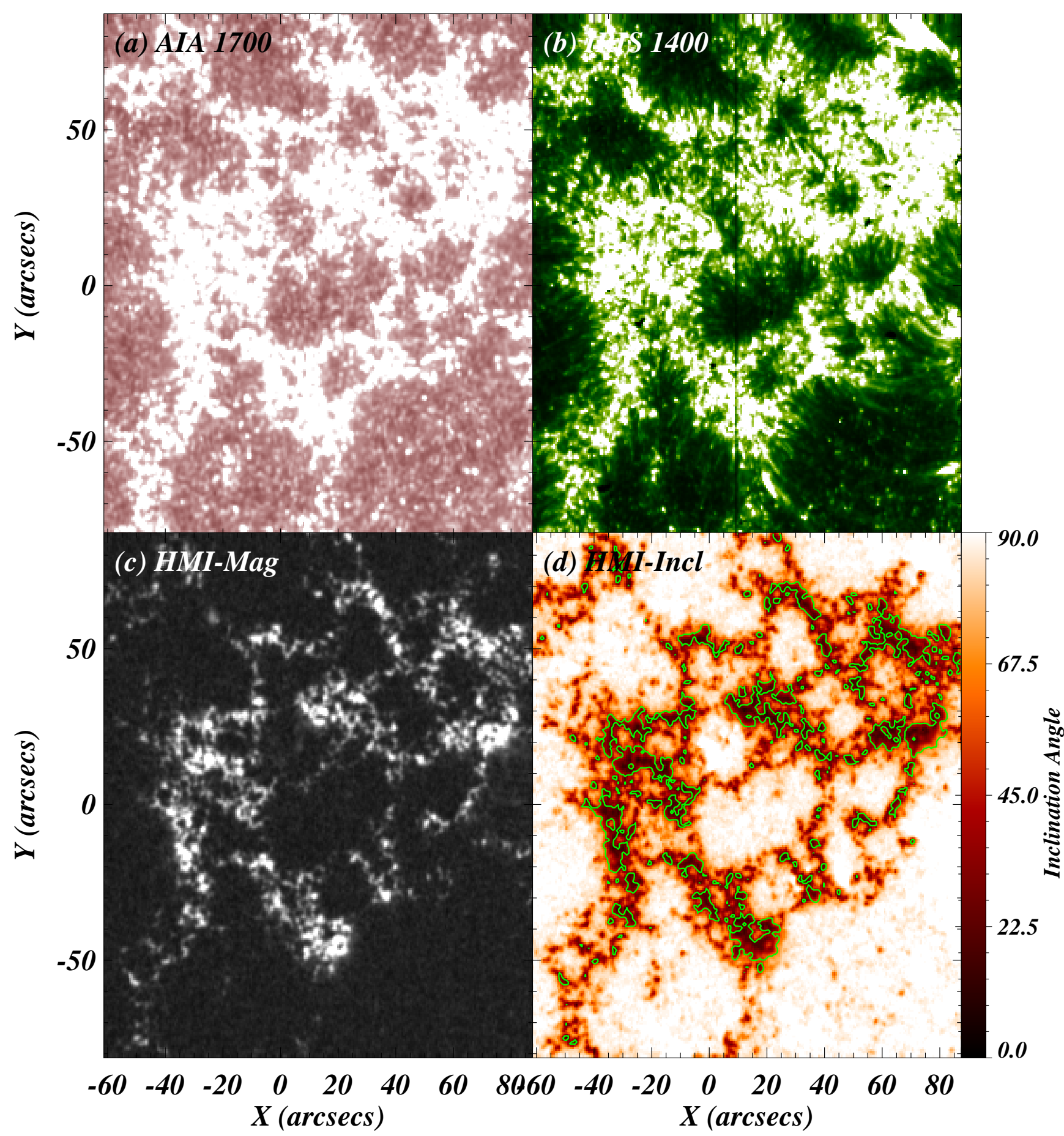

Fig. 1. Photospheric (SDO/AIA $1700 \AA$; panel $a$ ) and TR (IRIS SJI $1400 \AA$; panel $b$ ) images of the ROI (plage region). The plage is seen as highintensity patches clearly visible in the photosphere and in TR (panels $a$ and $b$ ). We show the absolute LOS magnetic field (panel $c$ ) and magnetic field inclination map (panel d) of the same region using SDO/HMI observations. A high magnetic field and low inclination angles are visible in the vicinity of the plage area. The plage locations were selected using a threshold of absolute magnetic field (i.e., above 300 gauss) and locations are contoured (i.e., above 300 gauss) over magnetic field inclination (panel $d$ ) in green, which reveal the presence of almost vertical (or very low inclination) fields.

the plage and the surrounding area at both heights in the solar atmosphere (e.g., AIA $1700 \AA$ and IRIS $1400 \AA$ ). Therefore, we observe that the behavior of the plage and surrounding region is almost the same at this high frequency. However, it should be noted that the power is very low at the photosphere (panel A) and TR (panel B). Furthermore, TR power is slightly lower than the photospheric power.
Intermediate frequency. The middle row of Fig. 2 shows the Fourier power maps of the photosphere (panel C) and TR (panel D) for intermediate frequency (i.e., $5.31 \mathrm{mHz}$ ). Specifically, this is in the frequency regime where most of the wave power is inherited and propagates into the higher layers. The pattern of photospheric wave power at intermediate frequency is completely different from the behavior that we found at high 

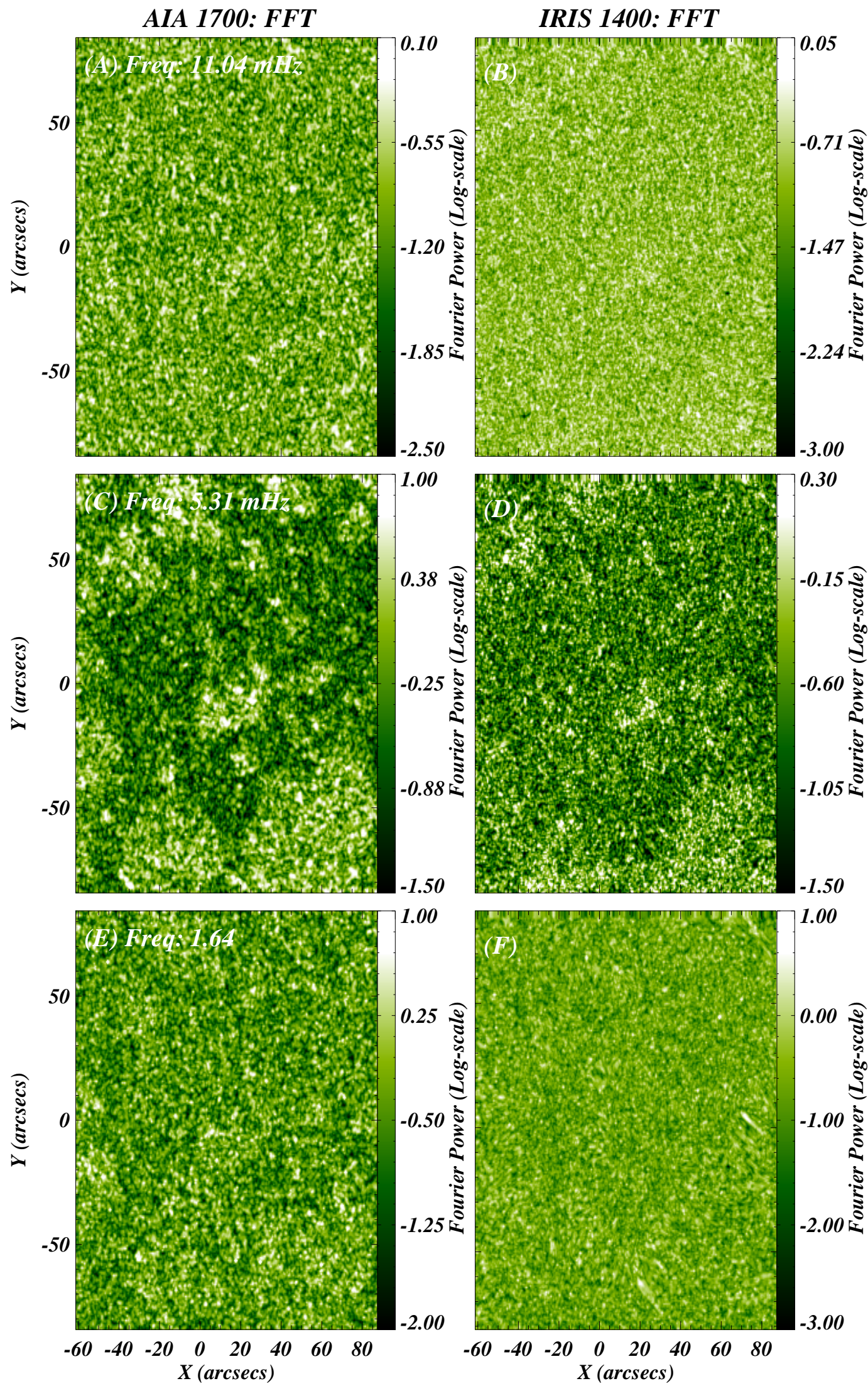

Fig. 2. Fourier power maps (log-scale) within the different frequency ranges for AIA $1700 \AA$ (left column) and IRIS $1400 \AA$ (right column). Top row: Fourier power map in the high-frequency range (i.e., $11.04 \mathrm{mHz}$ ) for the photosphere (panel $A$ ) and TR (panel B). Middle row: Fourier power maps in intermediate frequency (i.e., $5.37 \mathrm{mHz}$ ) for the photosphere (panel C) and TR (panel D). Bottom row: Fourier maps for low frequency (i.e., $1.64 \mathrm{mHz}$ ). 
frequencies. We see that power in the surrounding region is marginally higher than the inherited power in the plage. A similar behavior exists in the TR heights; however, the pattern is not as prominent as at the photospheric level.

Low frequency. The bottom row of Fig. 2 shows the Fourier power maps of the photosphere (panel E) and TR (panel F) at low frequency (i.e., $1.64 \mathrm{mHz}$ ). At this frequency range we do not see any difference between the plage and surroundings at the photospheric (panel E) and TR (panel F) levels.

\subsection{Wavelet analysis}

A wavelet transform involves the convolution between the TS and the "mother" function. There are different type of mother functions with the wavelet transform (e.g., Morlet, Paul, and derivative of Gaussian (DOG)). For the present analysis, we used the Morlet mother function with a dimensionless frequency $\omega_{0}=6$, which is suitable for investigating the propagation of waves in a range of frequencies. The Morlet function consists of a plane wave that is modulated by a Gaussian window:

$\psi_{0}(\eta)=\pi^{-1 / 4} \mathrm{e}^{i \omega_{0} \eta} \mathrm{e}^{-\eta^{2} / 2}$

Equation (1) describes the Morlet function, where $\omega_{0}$ and $\eta$ are the nondimensional frequency and time parameters. The wavelet transform provides a 2D complex array for a TS. The power of the wavelet is defined as the square of absolute magnitude of the complex wavelet. We show the intensity TS and wavelet power maps (i.e., $|W(s)|^{2}$ ) for the photospheric (panel a) and TR (panel c; Fig. 4) emission from a location within the selected plage locations. We did not apply any smoothing before the wavelet transform as it can add spurious periodicities in the TS (Auchère et al. 2016). We normalized the wavelet power as described in Sect. 3.1. A major fraction of the photospheric power is concentrated in periods shorter than $15 \mathrm{~min}$ (i.e., high frequency); however, significant power can also be found around $5 \mathrm{~min}$ and reflects the well-known photospheric oscillations (see Fig. 4). In the TR we also see the dominant power in high periods (panel d; Fig. 4). This power is not spurious and is persistent over long timescales (i.e., periods that exist for 25 min or longer, cf. Fig. 4). It indicates wave propagation from the photosphere into the TR. The white hatched area represents the cone of influence (COI) on each wavelet power map (cf. Fig. 4), while the solid white line outlines the $95 \%$ confidence levels.

The red or white noise spectrum is often used to calculate the confidence levels as described by Torrence \& Compo (1998). However, it should be noted that red or white noise does not really capture the inherited noise within the TS, which can in turn lead to an incorrect estimation of the confidence levels (Auchère et al. 2016). Ultraviolet (UV) and extreme ultraviolet (EUV) light curves tend to exhibit an overall power-law behavior, and the built-in red noise model is not appropriate to fit the spectrum (unless the power-law exponent is -2.0 ; priv. comm. with De Pontieu). In the present analysis, we also found that the TS shows that a power-law behavior with a broad distribution of the exponent values and a specific value of the power-law exponent (i.e., -2) are rare. Therefore, we can say that the builtin red-noise model is not appropriate to estimate the confidence levels.

Recently, Auchère et al. (2016) proposed a generic noise model for an EUV TS (using AIA high-temperature filter observations) and also showed that smoothing can add artificial periodicities. After Auchère et al. (2016) and Threlfall et al. (2017) also adopted the same generic noise model for EUV TS under different physical conditions of solar atmosphere. Auchère et al. (2016) proposed that the generic noise model is a linear combination of the power law, kappa function, and white noise. It is shown that the coronal signals are well fitted through this model. The present work deals with the signal from the lower solar atmosphere (i.e., photosphere and TR); therefore, the noise characteristics may be different from the inherited noise in the coronal time series. We checked this and found that signals used in this work are best represented by a power law. Hence, we utilized only a power-law function and omitted the kappa function and white noise contribution. We fitted a $\sigma(v)$ function to each power spectrum, which is described as follows:

$\sigma(v)=A v^{s}+C$.

Although the contribution from the constant ( $C$, i.e., white noise) is low in most of the conditions it may still play an important role in some cases. Therefore, we kept the constant term in our model. We first fitted the $\sigma(v)$ of each photospheric and TR power spectrum. Two samples are shown in Fig. 3 with various components. Each panel of Fig. 3 shows the FFT power spectrum, the time averaged wavelet spectrum, and the fitted generic noise model $(\sigma(v))$. Using this noise model, we estimated the local (blue line in both panels of Fig. 3) and global 95\% Fourier confidence level (pink line in both panels of Fig. 3). We also estimated the global $95 \%$ confidence levels which are shown by the gray solid line in both panels of Fig. 3. It should be noted that the global 95\% Fourier confidence level has high values compared to the local and global $95 \%$ wavelet confidence level for all periods. The local and global $95 \%$ confidence levels lie below the power spectra (i.e., Fourier and time-averaged wavelet) for most of the period bins. However, the global $95 \%$ wavelet confidence levels are higher than the local 95\% wavelet confidence level. It should be noted that similar findings are reported in previous works for coronal signals (Auchère et al. 2016; Threlfall et al. 2017). The estimated values of various parameters (e.g., $A, s$, and $\chi^{2}$ ) are also provided in each figure. The low $\chi^{2}$ values justify the reliability of the fitting model. Panels a and c are dedicated to AIA 1700, while panels b and d are for IRIS $1400 \mathrm{TS}$. Using the described methodology, we estimated wavelet power maps for AIA $1700 \AA$ and IRIS $1400 \AA$ and associated 95\% confidence levels (i.e., local and global wavelet, global Fourier) in the plage region. Figure 4 shows the normalized wavelet power map with the $95 \%$ wavelet global confidence level as white lines (panels $b$ and $d$ ). It shows that power is inherited over a broad range of periods within the photosphere and TR.

Furthermore, the wavelet coherence analysis is performed using AIA $1700 \AA$ and IRIS $1400 \AA$, which is important in order to understand coherent and incoherent oscillations at two different heights. In the very first step, the cross-spectrum (cross wavelet power) of two TS (photospheric and TR) is determined by multiplying the wavelet of one TS with the complex conjugate of the wavelet of another TS. The cross-spectrum highlights the power areas in the time-frequency range, which has common power in both TS. However, it should be noted that the cross-spectrum does not necessarily possess very high power as it is visible in the individual TS. The left panel of Fig. 6 shows the normalized cross-spectrum, which is drawn using the intensity TS of photosphere and TR. We utilized the cross FFT power and time-averaged cross wavelet power to estimate the 95\% global confidence levels for this cross wavelet power, which is further normalized by noise arrays (as described in Sect. 3.1). We adopted a similar methodology as we applied for individual TS. Figure 5 shows two different samples for estimation of the 

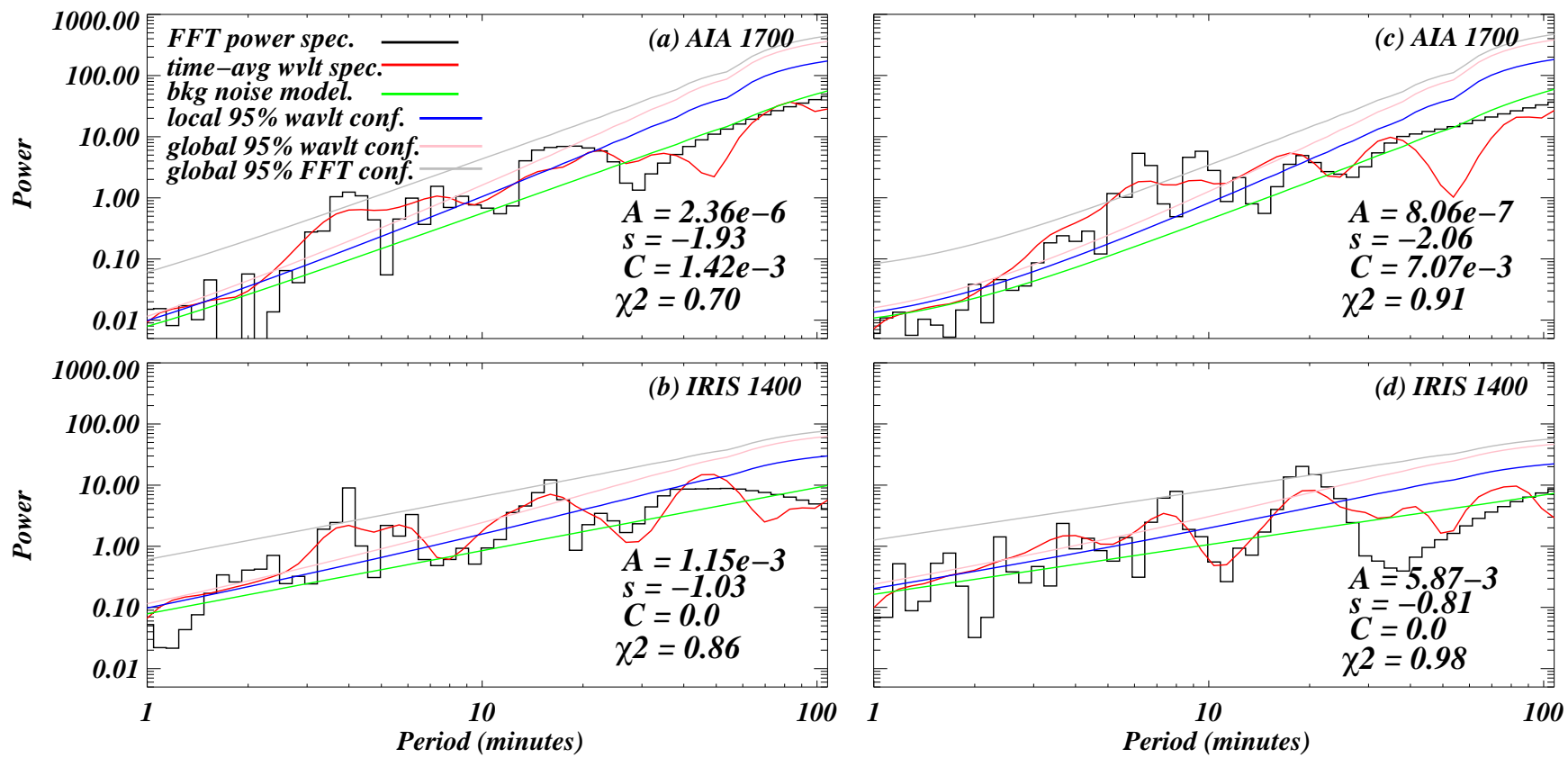

Fig. 3. Generic noise model for AIA 1700 (panels $a$ and $c$ ) and IRIS SJI 1400 (panels $b$ and $d$ ). The FFT power spectrum (black line), time averaged wavelet spectrum (red line), and fitted noise model (green line) are shown in each panel. Local and global 95\% confidence levels with fitted noise are shown in blue and pink, respectively. The parameters used for the fits are also provided. The low $\chi^{2}$ values justify the reliability of the fitted noise model on the FFT power spectrum.
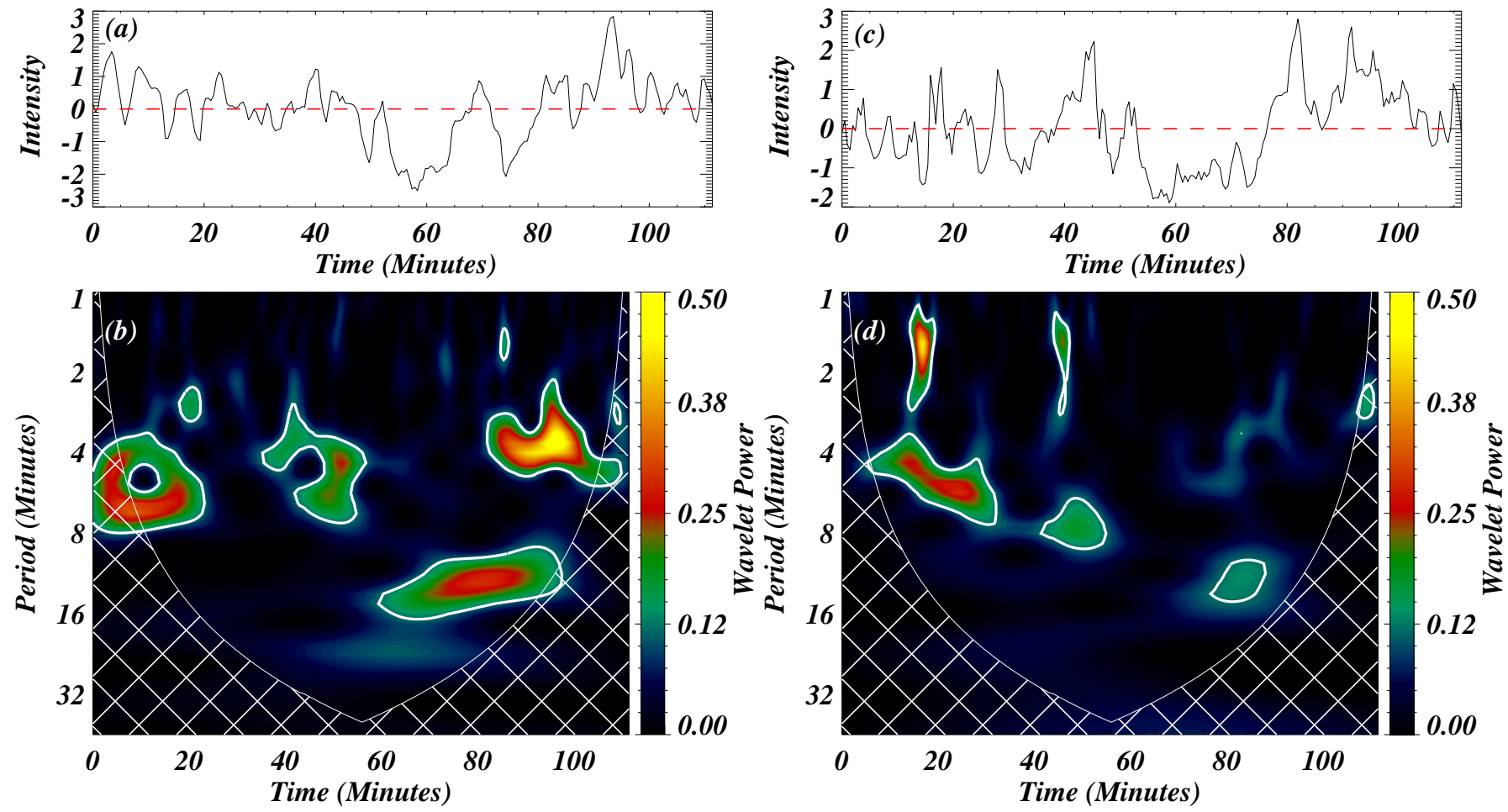

Fig. 4. Panel $a$ : AIA $1700 \AA$ TS. Panel $b$ : corresponding normalized wavelet power. The photospheric power is mainly concentrated in the broad range (2-9 min), as outlined by white contours (95\% global confidence levels). Panels $c$ and $d$ : IRIS SJI $1400 \AA$ TS and its corresponding normalized wavelet power, respectively. The power within the TR is distributed within the period of $2-8 \mathrm{~min}$. The white contours in each wavelet map (panels $b$ and $d$ ) outline the $95 \%$ global confidence levels. The cross-hatched white area outlines the COI.

95\% local and global confidence levels. The white line outlines the normalized cross wavelet power, which is above the $95 \%$ confidence level. The normalized cross wavelet power shows that a significant common power lies in the very wide range of periods, which is a signature of possible relation between the photosphere and the TR. However, it should be noted that common cross power alone is not sufficient in order to draw definite conclusions about wave propagation.

Therefore, we also studied the wavelet coherence, which is an important parameter used to investigate the interaction 

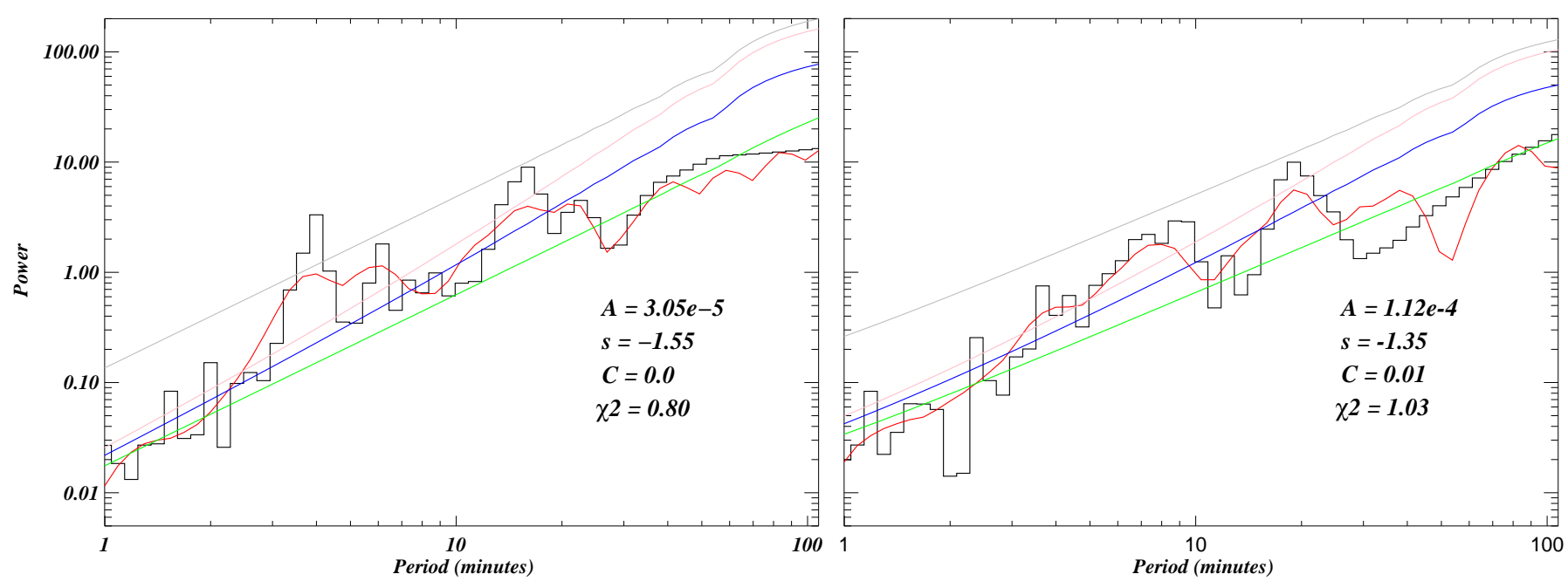

Fig. 5. Similar to Fig. 3, but for cross-spectra drawn using IRIS SJI $1400 \AA$ and AIA $1700 \AA$.

between two TS. The cross wavelet power is normalized by the multiplication of the power of both series, which is basically the wavelet coherence (Torrence \& Compo 1998). The coherence can vary from zero to one. A zero value represents complete incoherence and a value of one the perfect coherence between the two TS. The cross-spectrum of the TS is necessary to find significant common power in the time and frequency domains; however, the wavelet coherence is also needed to find the relationship between the two heights in the solar atmosphere. Therefore, wavelet coherence is an important parameter to signify the correlations of oscillations between two heights. The right panel of Fig. 6 shows the corresponding coherence map. Similar to the normalized cross power map, the coherence map also outlines $95 \%$ confidence levels by white lines. In addition, the hatched white area outlines the COI area on the cross power and coherence maps.

Finally, we estimated the phase lag (i.e., the difference in phase angles at two different heights) in the time and period domains. The cross wavelet gives the complex array in the time-period domain, which can be converted into the phase angle using real and imaginary parts of the complex numbers (Bloomfield et al. 2004; Jafarzadeh et al. 2017). The phase angle reflects the phase lag between the two intensity TS originating from two different heights. We used some specific conditions to select the valid phase lags for greater reliability of the results; for example, (a) only those regions that have significant cross power (lying within the regime of $95 \%$ global confidence level) were used, (b) the coherence value should be greater than 0.6 in these significant cross power areas, and (c) the COI area was excluded to avoid spurious edge effects. Therefore, we extracted the valid phase lags using these conditions in the time-period domain. We used arrows to show these valid phase lags on the time-period domain. These valid phase lags are overplotted on the coherence maps using white (positive phase lag) and black arrows (negative phase lags). The positive phase lags indicate the upward propagation, while the negative phase lags represent the downward propagation of the waves between two heights. The phase lag is positive in the short-period regime (i.e., less than $6.0 \mathrm{~min}$ ), which shows the propagation of the waves from the photosphere to the TR (right panel; Fig. 6). However, a negative phase lag is evident within the long-period regime (i.e., greater than $8.0 \mathrm{~min}$ ). This observational finding indicates that the waves within the period range of 2.0-6.0 min propagate upward from the photosphere and can reach up to the TR.
We applied the same procedure to all the plage locations to extract valid phase lags. After extracting the phase lags we produced the 2D histogram (i.e., phase lag versus period) using all plage locations. Panel a of Fig. 7 shows a 2D histogram of phase lags. The histogram reveals the presence of significant phase lag within the period range from 2.0 to $9.0 \mathrm{~min}$. Periods of less than $7.0 \mathrm{~min}$ are dominated by the positive phase angles, while for periods longer than $7.0 \mathrm{~min}$ the phase angles are distributed both in the positive and negative regimes. The overplotted blue contours are fitted 2D Gaussian, which successfully characterize the behavior of the observed phase lag distribution. With the help of 2D Gaussian fitting, we estimated the peak phase angle at every period. Panel $b$ in Fig. 7 shows the distribution of phase angles at a period of $4.35 \mathrm{~min}$. At this period the peak of the distribution lies around $43.56^{\circ}$ with the $1 \sigma$ spread of $39.94^{\circ}$ (see panel $b$ ). Finally, we produced the behavior of the mean phase lag with the periods shown in panel c of Fig. 7. The phase lag decreases with increasing period. It is clearly visible that zero phase angle occurs around $9.0 \mathrm{~min}$ (cf. panels c and a), which predicts that waves with periods of less than 9.0 min successfully propagate from the photosphere into the TR.

\subsection{Evidence of the propagation of magneto-acoustic waves}

In this work our aim is to investigate the propagation of waves within VMPL through phase difference $(\Delta \phi)$. The phase difference between the photospheric (AIA 1700) and TR (IRIS SJI $1400 \AA$ ) reveals the presence of a positive phase lag within the period regime from 2.0 to $9.0 \mathrm{~min}$ (cf. Fig. 7). The value of $\Delta \phi$ decreases with the period. This observational finding suggests that most of the photospheric power leaks into the TR through the wave propagation in this period regime. We performed some further investigations to understand the nature of these waves. Using $\Delta \phi$ and the corresponding frequency and height difference between the two atmospheric layers, we are able to estimate the wave travel time and propagation speed. For instance, $\Delta \phi$ is around $63.20^{\circ} \pm 34.90^{\circ}$ at the frequency of $5.4 \mathrm{mHz}$ (or around $3.0 \mathrm{~min}$; cf. Fig. 7):

$\tau=\frac{\Delta \phi}{2 \pi f}$

Using Eq. (3) and the upper value of $\Delta \phi(\mu+2.0 \sigma)$, the wave travel time $(\tau)$ is around $68.34 \mathrm{~s}$ for the frequency of $5.4 \mathrm{mHz}$. 

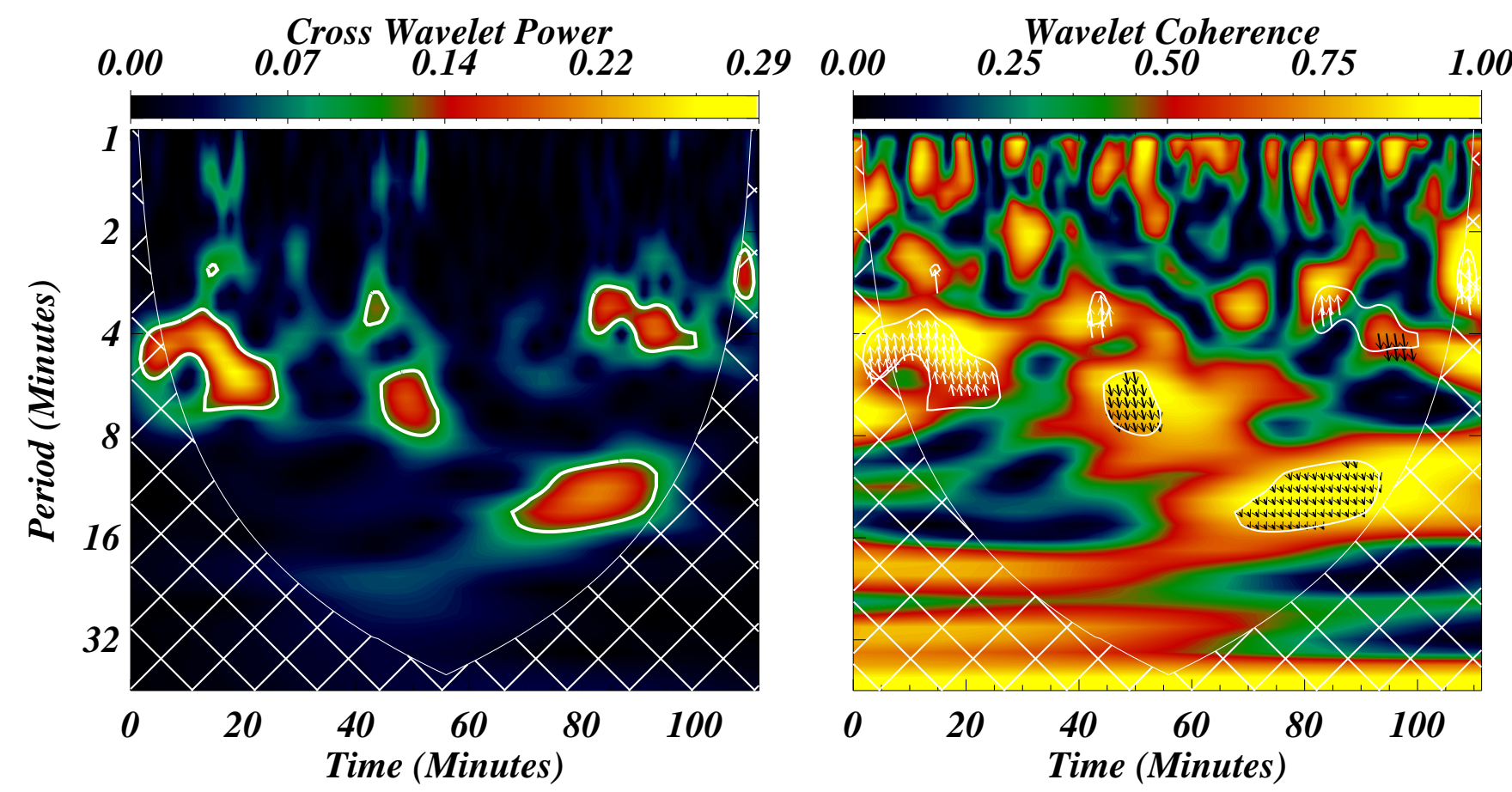

Fig. 6. Left panel: normalized cross wavelet power between AIA $1700 \AA$ and IRIS SJI $1400 \AA$. The normalized cross power is distributed in a period range similar to that in the case of wavelet power of photosphere and TR (cf. Fig. 4). Right panel: wavelet coherence is displayed along with the overplotted phase difference angle (black and purple arrows) within the high-coherence regime (above 0.6). Negative phase difference (black arrows) represent downward propagating waves, while positive phase difference (purple arrows) indicates upwardly propagating waves. It is clearly visible that below $7.0 \mathrm{~min}$ the waves are propagating upward into the TR from the photosphere. The white contours outline the $95 \%$ confidence levels, while the white cross-hatched area outlines the COI.

The Si IV $1393.77 \AA$ spectral line typically forms at a geometrical height of $2-3 \mathrm{Mm}$ above the photosphere. Let us assume a height difference of $2 \mathrm{Mm}$ (lower bound value) between the photosphere (AIA $1700 \AA$ ) and TR (IRIS SJI $1400 \AA$ ). This means that the wave travel time and distance (height difference between photosphere and TR) yield a wave propagation speed of $\sim 29 \mathrm{~km} \mathrm{~s}^{-1}$. It is believed that waves travel at slightly lower speed (i.e., 15-20 $\mathrm{km} \mathrm{s}^{-1}$ ) within the lower solar atmosphere (B. De Pontieu, priv. comm.) and our estimated propagation speed is higher than expected. We used VMPLs (very bright and highmagnetic field areas) that can have significantly higher temperatures than the QS. We do not have the temperature measurement at the present baseline, although qualitatively we can assume that plages should have higher temperatures than the QS. These higher temperatures can lead to a higher sound speed, so we can infer that the speed of the propagating waves is close to the sound speed within the plage region. Our observational findings indicate that these are SMAWs. De Pontieu et al. (2003, 2005) also report the propagation of SMAW into the corona from the photosphere in plage regions. Nonlinear effects contribute to complex wave dynamics (Heggland et al. 2011; Skogsrud et al. 2016), while the occurrence of shocks produce strong gradients in various physical parameter (i.e., intensity or Doppler velocity) over a short period of time (Tian et al. 2014). Variations in the physical parameters (i.e., intensity or Doppler velocity) should reveal the saw-tooth pattern reported by Tian et al. (2014). Panel b of Fig. A. 1 shows the Si IV $1393.77 \AA$ intensity images $(y-t$ images).

A large number of samples (5927 TS within the plage) are utilized in the present analysis. We find that a large number of locations show good correlation between the photosphere and the TR over a significant time interval, and is the reason why we used all the locations to create the final 2D histogram (cf. Fig. 7). Previous reports state that only a small fraction of the locations show a nice correlation between two atmospheric layers. For example, De Pontieu et al. (2003) report that only 4\% of the locations (6 out of the total 148) show a good correlation that indicate the successful propagation of the SMAW between the photosphere and TR. In the present observation, we find that almost all locations have a correlation between the photosphere and TR. In previous work, with the help of FFT, Centeno et al. (2009) utilize all the locations to draw the phase difference diagram (frequency versus phase) between photosphere and chromosphere. We also used all the locations to draw the phase versus frequency histogram. In this work we used IRIS highresolution observations and analysis techniques (wavelet, crossspectrum, coherence, and a generic noise model), which lead to these results with even more accuracy. A higher occurrence of propagation of SMAW within the plage region is inferred from IRIS observations for the first time. We believe that this is important for energy transport, and thus the possible heating of the atmosphere overlying the plage region.

\section{Discussion and conclusions}

Centeno et al. $(2006,2009)$ investigated wave propagation in different features of the solar atmosphere (e.g., umbra, pore, and faculae) through $\Delta \phi$ using FFT. They reported a successful propagation of $3 \mathrm{~min}$ oscillations into the chromosphere for each of these solar features. In the case of faculae, they found that low-frequency waves $(5 \mathrm{~min})$ can also reach the chromosphere. The variation of cut-off frequency was also investigated using frequency versus $\Delta \phi$ diagrams (Centeno et al. 2006, 2009). Their findings suggest that waves with longer periods can also 

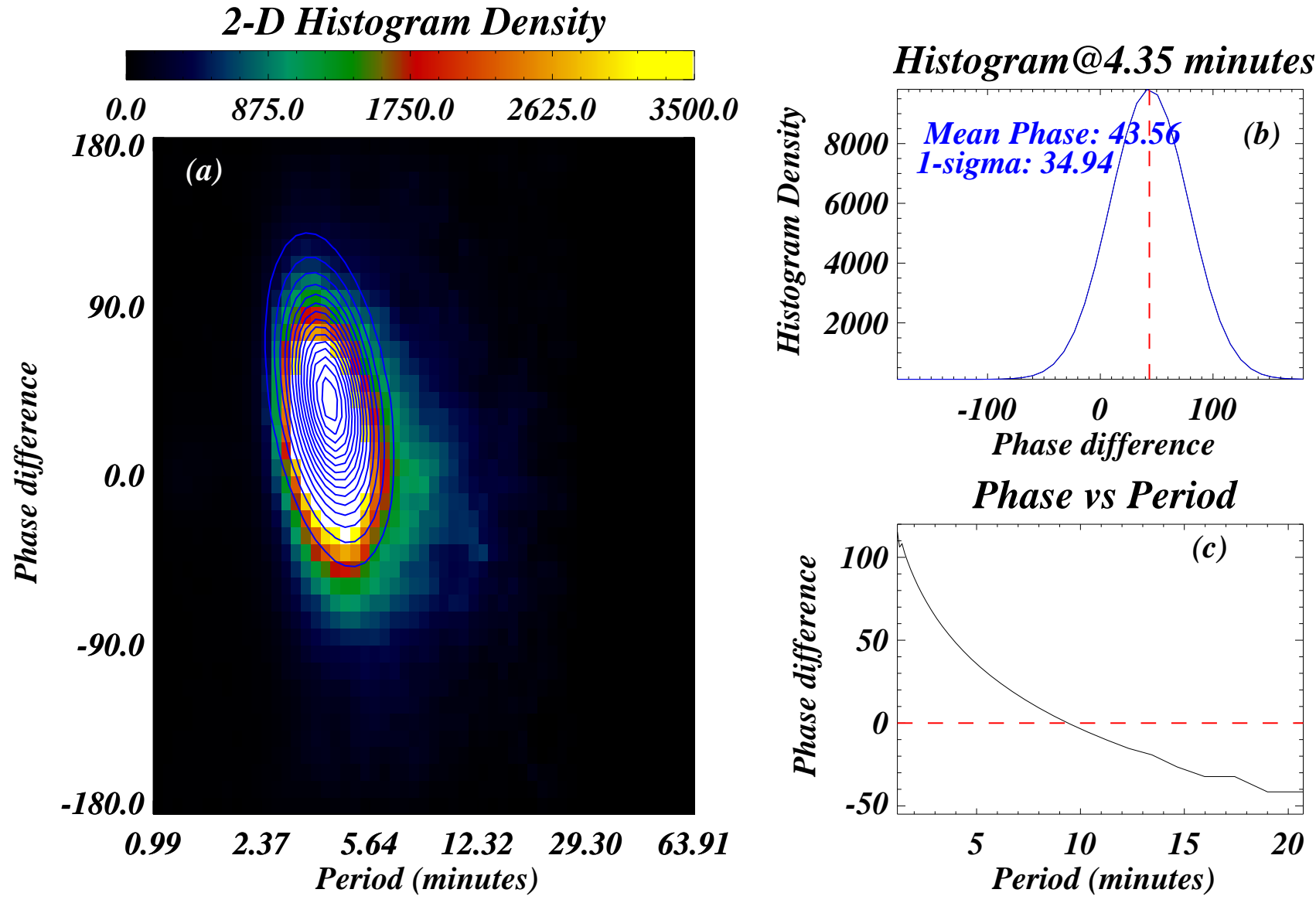

Fig. 7. Panel a: two-dimensional histogram of $\Delta \phi$, extracted from all plage locations. The white contours show a fitted 2D Gaussian on the 2D $\Delta \phi$ histogram. The histogram reveals that shorter periods have positive phase difference, while longer periods move into the negative phase difference regime. With the help of a 2D Gaussian, the peak phase difference was estimated for each period. Panel $b$ : distribution of phase difference at the period of $4.35 \mathrm{~min}$. Panel $c$ : phase difference as a function of period. A vertical dashed line in panel $b$ indicates the position of peak phase difference. A dashed horizontal line in panel $c$ indicates zero phase difference.

propagate into the TR in a plage environment. Centeno et al. (2006, 2009) used only vertical magnetic field and successfully managed to show the propagation of $5 \mathrm{~min}$ oscillations in faculae. De Pontieu et al. (2004) reported that the leakage of $p$-modes into the upper atmosphere is only possible along inclined magnetic fields, while Centeno et al. (2009) reported the propagation of $5 \mathrm{~min}$ waves in faculae for non-inclined (almost vertical) magnetic field. Heggland et al. (2011) used numerical simulations to study wave propagation. Waves with a $5 \mathrm{~min}$ period dominate in strong and inclined flux-tubes, whereas 3 min dominates in weak or vertical magnetic flux tubes. Similarly to De Pontieu et al. (2004) and Heggland et al. (2011) also found that flux tube inclination is important for long-period wave propagation. Furthermore, De Pontieu et al. (2003, 2005) reported that longer period waves associated with the photospheric $5 \mathrm{~min}$ oscillations can reach into the TR within the plage region.

In the present work we estimated 2D Fourier power maps from plage observations (i.e., plage plus the surrounding area) at different frequency ranges (from high to low frequencies). The Fourier power maps show how the wave power is distributed in the plage and surrounding area and show that most of the wave dynamics are inherited in the intermediate frequency range. We found very low power at high frequencies without any significant difference in the plage and surrounding region. Similarly, low frequencies do not show the difference between the plage and surrounding region, but have high power in comparison to the power inherited in the high-frequency range. Hence, on average, the Fourier power behavior suggests that the dynamics of the plage region is inherited within the regime of intermediate frequencies. Then we applied for the first time a generic noise model to estimate the confidence levels using wavelet analysis on photospheric and TR TS. The noise model was previously applied only for coronal TS (Auchère et al. 2016; Threlfall et al. 2017). Previous scientific works utilized either a white or red noise model to calculate confidence levels, which can give erroneous confidence levels. In addition, Auchère et al. (2016) reported that smoothing can include spurious periods; therefore, we did not apply any type of smoothing to our TS. The generic noise model and original TS significantly improved the accuracy of the results. Our analysis reveals that waves within a certain period range $(2.0-9.0 \mathrm{~min})$ are propagating into the TR from the solar photosphere. We find that $\Delta \phi$ decreases with period, which qualitatively matches the previous reported results (e.g., Centeno et al. 2006, 2009).

Using $\Delta \phi$, frequency, and the height difference (between photosphere and TR) we estimated the wave travel time and propagation speed as $68.34 \mathrm{~s}$ at a frequency of $5.4 \mathrm{mHz}$, which gives a propagation speed of about $29.26 \mathrm{~km} \mathrm{~s}^{-1}$. This propagation speed is close to the sound speed in the TR, which justifies our conclusion that these are SMAWs. In addition, a significant correlation or propagation (in terms of the locations) between the photosphere and TR is revealed in the present analysis for 
the first time using IRIS and AIA observations. Previous works report a very small fraction of good correlation between photosphere and TR (De Pontieu et al. 2003). The occurrence frequency of correlation or propagation of SMAW is very high in the present study. It should be noted that we only used the almost vertical magnetic field plage locations and find that a broad range of waves (from 2 to 9 min) successfully reach into the TR from photosphere. Finally, our results suggest that the propagation of 5 min oscillations may not depend on the magnetic field inclination, which supports the findings of Centeno et al. (2006, 2009). We investigated the nonlinear character of waves (i.e., shocks) and did not find any such signature.

We investigated which emission (i.e., photospheric - continuum part of SJI $1400 \AA$ filter or Si IV lines in SJI $1400 \AA$ ) dominates in the SJI $1400 \AA$. On the basis of a light curve comparison and phase analysis, we find that the SJI $1400 \AA$ emission is dominated by the Si IV lines (see Appendix A for more details). Similarly, Skogsrud et al. (2016) report that bright grain emission originates from the TR (i.e., SiIV lines) rather than the photosphere (i.e., continuum part of SJI 1400 filter). However, using the same observations, Martínez-Sykora et al. (2015) suggests that the emission of bright grains originates from the photosphere.

Acknowledgements. We sincerely thank Dr. Clara Froment for her constructive comments that improved the paper significantly. We would like to thank Dr. Bart De Pontieu (LMSAL) for helpful comments on a previous version of the paper. IRIS is a NASA small explorer mission developed and operated by LMSAL with mission operations executed at NASA Ames Research center and major contributions to downlink communications funded by ESA and the Norwegian Space Centre. S.K.T. gratefully acknowledges support by the NASA contract NNG09FA40C (IRIS). AKS and MM acknowledge the support of the UGCUKIERI grant to support the present research.

\section{References}

Auchère, F., Bocchialini, K., Solomon, J., \& Tison, E. 2014, A\&A, 563, A8 Auchère, F., Froment, C., Bocchialini, K., Buchlin, E., \& Solomon, J. 2016, ApJ, 825,110

Bloomfield, D. S., McAteer, R. T. J., Lites, B. W., et al. 2004, ApJ, 617, 623
Cally, P. S. 2007, Astron. Nachr., 328, 286

Cally, P. S., Bogdan, T. J., \& Zweibel, E. G. 1994, ApJ, 437, 505

Centeno, R., Collados, M., \& Trujillo Bueno, J. 2006, ApJ, 640, 1153 Centeno, R., Collados, M., \& Trujillo Bueno, J. 2009, ApJ, 692, 1211

De Moortel, I., Ireland, J., \& Walsh, R. W. 2000, A\&A, 355, L23

De Moortel, I., Ireland, J., Hood, A. W., \& Walsh, R. W. 2002, A\&A, 387, L13

De Pontieu, B., Erdélyi, R., \& de Wijn, A. G. 2003, ApJ, 595, L63

De Pontieu, B., Erdélyi, R., \& James, S. P. 2004, Nature, 430, 536

De Pontieu, B., Erdélyi, R., \& De Moortel, I. 2005, ApJ, 624, L61

De Pontieu, B., Title, A. M., Lemen, J. R., et al. 2014, Sol. Phys., 289, 2733

Falconer, D. A., Tiwari, S. K., Moore, R. L., \& Khazanov, I. 2016, ApJ, 833, L31

Froment, C., Auchère, F., Bocchialini, K., et al. 2015, ApJ, 807, 158

Gabriel, A. H., Baudin, F., Boumier, P., et al. 2002, A\&A, 390, 1119

Gupta, G. R. 2014, A\&A, 568, A96

Heggland, L., Hansteen, V. H., De Pontieu, B., \& Carlsson, M. 2011, ApJ, 743, 142

Inglis, A. R., Ireland, J., \& Dominique, M. 2015, ApJ, 798, 108 Ireland, J., McAteer, R. T. J., \& Inglis, A. R. 2015, ApJ, 798, 1

Jafarzadeh, S., Solanki, S. K., Stangalini, M., et al. 2017, ApJS, 229, 10

Jelínek, P., \& Karlický, M. 2009, Eur. Phys. J. D, 54, 305

Jelínek, P., \& Murawski, K. 2013, MNRAS, 434, 2347

Jelínek, P., Srivastava, A. K., Murawski, K., Kayshap, P., \& Dwivedi, B. N. 2015, A\&A, 581, A131

Jess, D. B., De Moortel, I., Mathioudakis, M., et al. 2012, ApJ, 757, 160

Jess, D. B., Morton, R. J., Verth, G., et al. 2015, Space Sci. Rev., 190, 103

Judge, P. G., Tarbell, T. D., \& Wilhelm, K. 2001, ApJ, 554, 424

Kayshap, P., Murawski, K., Srivastava, A. K., Musielak, Z. E., \& Dwivedi, B. N. 2018, MNRAS, 479, 5512

Khomenko, E., \& Collados, M. 2015, Liv. Rev. Sol. Phys., 12, 6

Krishna Prasad, S., Jess, D. B., \& Khomenko, E. 2015, ApJ, 812, L15

Lemen, J. R., Title, A. M., Akin, D. J., et al. 2012, Sol. Phys., 275, 17

Lites, B. W., Chipman, E. G., \& White, O. R. 1982, ApJ, 253, 367

Martínez-Sykora, J., Rouppe van der Voort, L., Carlsson, M., et al. 2015, ApJ, 803,44

Murawski, K., Kayshap, P., Srivastava, A. K., et al. 2018, MNRAS, 474, 77

Pugh, C. E., Broomhall, A.-M., \& Nakariakov, V. M. 2017, A\&A, 602, A47

Skogsrud, H., Rouppe van der Voort, L., \& De Pontieu, B. 2016, ApJ, 817, 124

Spruit, H. C. 1991, in Challenges to Theories of the Structure of Moderate-Mass

Stars, eds. D. Gough, \& J. Toomre (Berlin: Springer Verlag), Lect. Notes Phys., 388, 121

Srivastava, A. K., Kuridze, D., Zaqarashvili, T. V., \& Dwivedi, B. N. 2008, A\&A, 481, L95

Threlfall, J., De Moortel, I., \& Conlon, T. 2017, Sol. Phys., 292, 165

Tian, H., DeLuca, E., Reeves, K. K., et al. 2014, ApJ, 786, 137

Torrence, C., \& Compo, G. P. 1998, Bull. Am. Meteorol. Soc., 79, 61

Vaughan, S. 2005, A\&A, 431, 391

Wikstøl, Ø., Hansteen, V. H., Carlsson, M., \& Judge, P. G. 2000, ApJ, 531, 1150 


\section{Appendix A: Consistency between IRIS SJI $1400 \AA$ and Si iv $1393.77 \AA$}

As we derive well-correlated oscillations in near photospheric (AIA $1700 \AA$ ) and TR (IRIS SJI $1400 \AA$ emission), we need to evaluate whether the IRIS SJI $1400 \AA$ bandpass is indeed dominated by the TR emission. The IRIS SJI $1400 \AA$ filter is a $55 \AA$ broad filter, which contains two strong Si IV lines. However, a large portion of this filter is dominated by the photospheric continuum. The Si IV $1393.77 \AA$ spectral line, which is the strong line within this filter, is also included for this observation. With the help of this spectral line, we can check the dominance of emissions within this filter.

First, we estimated the intensities from the Si IV $1393.77 \AA$ line using Gaussian fitting. By selecting the slit position in SJ images, we produced $y$ (along the slit)-t(time) intensity images from IRIS SJI $1400 \AA$ data cube. This allows a comparison between the $y-t$ intensity images of SJI $1400 \AA$ and Si IV $1393.77 \AA$. The intensity image from SJI $1400 \AA$ ) is very similar to the intensity image from Si IV $1393.77 \AA$ (cf. panels a and b of Fig. A.1). We also examined TS from two different locations within the plage (cf. panels c and d of Fig. A.1). This shows that the SJI $1400 \AA$ emission exactly follows the TR emission (emissions from Si IV $1393.77 \AA$ ). Using intensity threshold, we divide the regions into plage and surrounding QS. We then look for correlations between the IRIS SJI $1400 \AA$ and Si IV $1393.77 \AA$ TS for each location. Panel c of Fig. A.1 shows the statistical distri- bution of correlation coefficients for plage (red histogram) and QS (black histogram). The histograms reveal that the QS coefficients show a very wide spread, while the plage histogram is sharply peaked at a high value (around 0.7). It is also evident that the mean coefficient is high for the plage region (0.75) compared to QS (0.6). These observational findings support that SJI $1400 \AA$ and Si IV $1393.77 \AA$ are highly correlated with each other in the plage regions.

In a further step, we investigated the phase difference between IRIS SJI $1400 \AA$ and Si IV 1393.77 $\AA$. The Si IV 1393.77 $\mathrm{A}$ line forms in the TR. If IRIS SJI $1400 \AA$ is dominated by the emission originating from the TR, then the phase lag between SJI $1400 \AA$ and Si IV $1393.77 \AA$ should be around zero phase. Applying the same methodology as previously described, we estimated the phase lag between SJI $1400 \AA$ and Si IV $1393.77 \AA$ and this is shown in Fig. A.2. The 2D histogram a shows that almost all phase angles are accumulated within \pm 15 in each period. In addition, the phase lags have no dependency on the periods, as is expected theoretically and as we found previously. In b we also show the statistical distribution of the estimated phase differences. The black line is a histogram of phase lags, while the overplotted blue line is the Gaussian fit of the histogram. The distribution shows that the peak lies around zero phase difference (i.e., -1.16). Moreover, the Gaussian fitting shows that the full width at half maximum (FWHM) is around 20. On the basis of these observational findings, we can say that IRIS SJI $1400 \AA$ captures the emissions from TR within the plage areas.
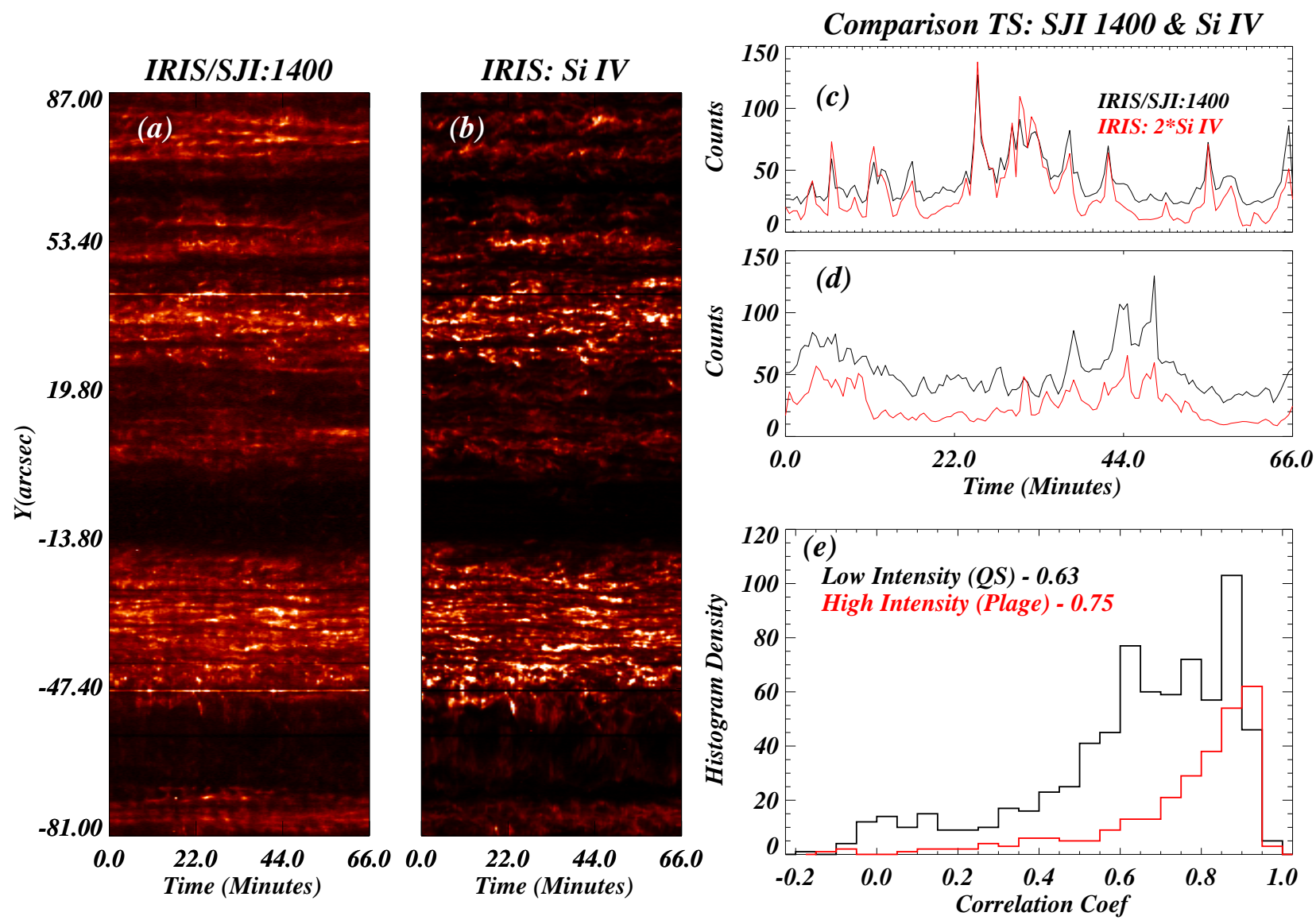

Fig. A.1. Panels $a$ and $b$ : $y-t$ intensity images from IRIS SJI $1400 \AA$ and Si IV $1393.77 \AA$. These images are very similar. We compared IRIS SJI $1400 \AA$ TS (black line) with Si IV $1393.77 \AA$ (two times Si IV) from two different locations (panels $c$ and $d$ ) to understand the similarities and differences in the nature of both TS. Panel e: histograms of the correlation coefficients for QS (black) and plage (red). The plage histogram is sharply peaked towards high values. 

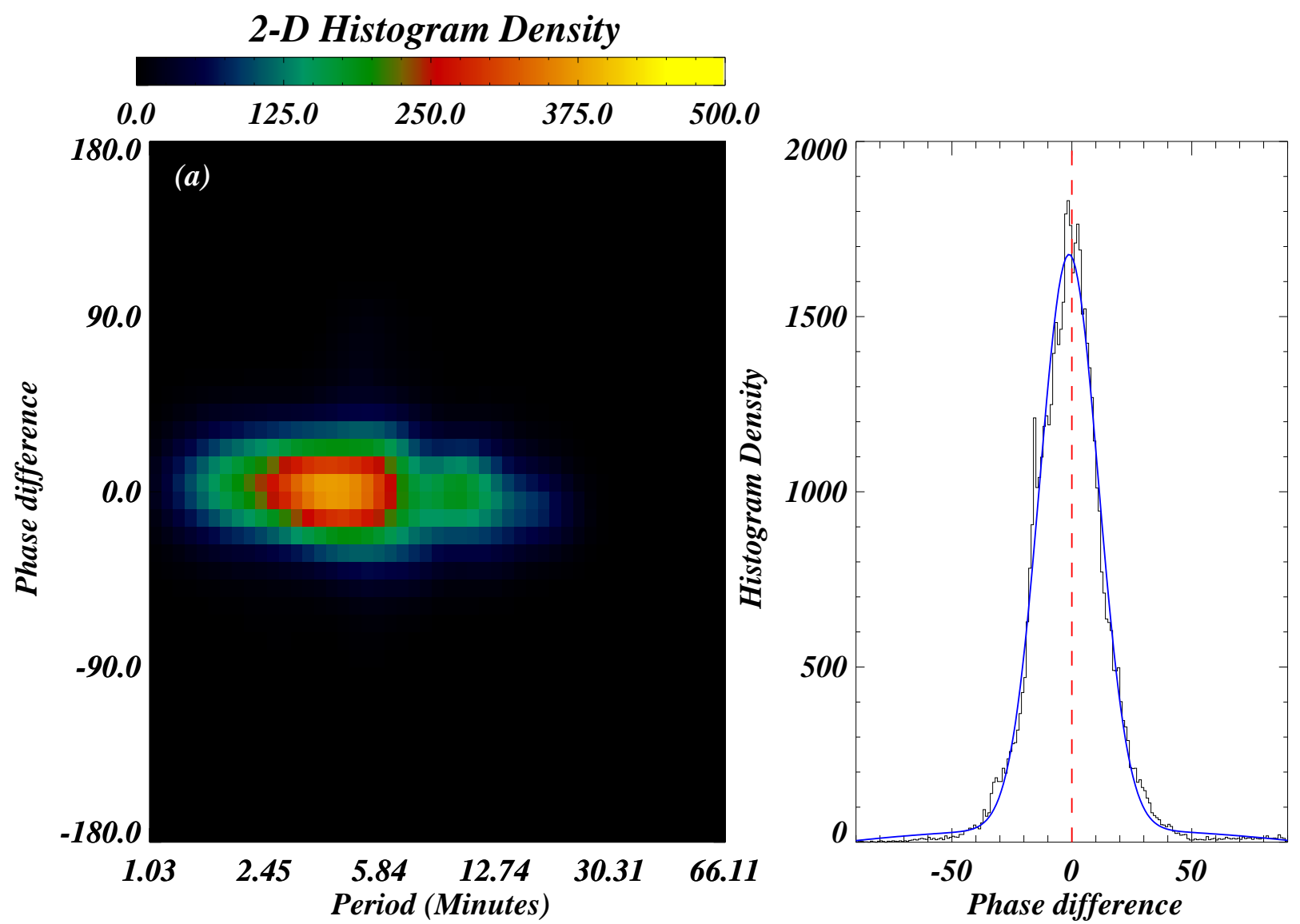

Fig. A.2. Panel $a$ : two-dimensional histogram of phase differences distributed within \pm 20 . Panel $b$ : distribution of phase difference (black line), estimated using IRIS SJI $1400 \AA$ and Si IV $1393.77 \AA$, along with its Gaussian fitting (blue line). The statistical distribution peaks around a 0 phase difference with a FWHM of 20. A vertical dashed line marks the location of zero phase difference. 